\title{
Note on a Schurer-Stancu-type operator
}

\author{
Adrian D. Indrea, ANAMARIA INdreA and Petru I. BraicA
}

\section{ABSTRACT.}

The aim of this paper is to introduce a class of operators of Schurer-Stancu-type with the property that the test functions $e_{0}$ and $e_{1}$ are reproduced. Also, in our approach, a theorem of error approximation and a Voronovskaja-type theorem for this operators are obtained. Finally, we study the convergence of the iterates for our new class of operators.

\section{REFERENCES}

[1] Agratini, O., An asymptotic formula for a class of approximation processes of King's type, Studia Sci. Math Hungar, 47 (2010), No 4, 435-444

[2] Agratini, O. and Rus, I., A., Iterates of a class of discrete linear operators via contraction principle, Comment. Math. Univ. Carolin., 44 (2003), $555-563$

[3] Bărbosu, D., Polynomial Approximation by Means of Schurer- Stancu type operators, Ed. Univ. de Nord Baia Mare, 2006

[4] Braica, P. I., Pop, O. T. and Indrea, A. D., About a King-type operator, Appl. Math. Inf. Sci, 6 (2012), No. 1, 191-197

[5] Braica, P.,I., Pop, O.,T., Bărbosu, D., Schurer operators of King type, Creative Math. Inf., 22 (2013), No. 2, 161-171

[6] Indrea, A. D., A particular class of linear and positive Stancu-type operators, Acta Univ. Apulensis, No. 31 (2012), 249-256

[7] King, J. P., Positive linear operators which preserve $x^{2}$, Acta Math. Hungar., 99 (2003), No. 3, 203-208

[8] Oancea, Ingrid A., Berstein - Stancu type operator wich preserves e e, An. Şt. Univ. Ovidius Constanţa, Vol. 17 (2009), No. 1, 137-144

[9] Pop, O. T., The generalization of Voronovskaja's theorem for a class of liniar and positive operators, Rev. Anal. Numer. Théor. Approx., 34 (2005), No. 1,79-91

[10] Pop, O. T., Bărbosu, D. and Braica, P. I., Berstein type operators preserving exactly two test functions, Studia Scient. Math. Hu., Vol. 50 (2013), No. 4, 393-405

[11] Schurer, F., Linear positive operators in approximation theory, Math. Inst. Techn. Univ. Delft Report, 1962

[12] Stancu, D. D., On a generalization of the Bernstein polynomials, Studia Univ. "Babeş - Bolyai", Scr. Math - Phis 14 (1969), 31-45 (in Romanian)

BABEŞ BOLYAI UNIVERSITY

FACUlTY OF MATHEMATICS AND COMPUTER SCIENCE

KOGĂLNICEANU 1, 400084 CLUJ-NAPOCA, ROMANIA

E-mail address: adrian.indrea@yahoo.com

BABEŞ BOLYAI UNIVERSITY

FACULTY OF MATHEMATICS AND COMPUTER SCIENCE

KOGĂLNICEANU 1, 400084 ClUJ-NAPOCA, ROMANIA

E-mail address: anamaria.indrea@yahoo.com

SCHOOL "GRIGORE MOISIL"

MileniUlui 1, 440037 SATU Mare,Romania

E-mail address: petrubreyahoo.com 